\title{
Le système \\ de santé chinois
}

Carine Milcent

> Côté santé, la Chine connaît une situation paradoxale, avec des indicateurs dont la courbe de progression est spectaculaire: une espérance de vie passée de 43,7 ans en 1960 à 76,9 ans en 2019 ; des dépenses de santé à hauteur de 5,35\% du produit intérieur brut (PIB) en $2018^{1} \ldots$ Mais un système décrit par de nombreux observateurs comme manquant d'efficacité et des relations excessivement tendues voire violentes $[1,2]$ entre, d'un côté, le personnel soignant et les médecins et, de l'autre côté, les patients et leur famille. Pour comprendre le système de santé chinois, je m'attacherai dans cette revue à le contextualiser puis je montrerai comment, face à l'incapacité à mettre en place un système fondé sur des modèles existants, ce système de santé est en train de se redessiner en développant un système alternatif de soins. <

\section{Pourquoi une formation à la médecine si disparate}

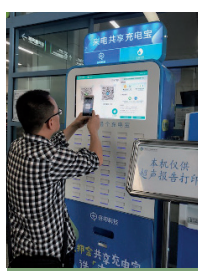

Chercheure CNRS, Professeure associée, CNRS UMR 8174, École d'économie de Paris (PSE Paris School of Economics), Campus Jourdan, 48 boulevard Jourdan, 75014 Paris, France. carine.milcent@psemail.eu

Nommés «médecins aux pieds nus » (chijiao yisheng), ces paysans apportaient des soins primaires et des actions de prévention de santé [5]. Ils avaient alors un titre de médecin même si leur qualification ne correspondait pas à celui d'un médecin hospitalier. L'objectif était de diffuser les rudiments de la médecine. Les patients étaient examinés afin de définir leurs besoins de soins. Cela permettait un premier tri des malades. En fonction de la sévérité des cas, le patient était redirigé vers des structures aux personnels plus qualifiés. Jusqu'aux années 1980, pour les habitants des zones rurales, le système de santé chinois était organisé en trois niveaux d'accès aux soins [6] : le dispensaire, dans le village; le centre de santé, dans le canton; et l'hôpital local, dans le district ${ }^{4}$. Une universalité d'accès aux soins et une quasigratuité étaient ainsi assurées, mais pour un niveau de qualité médiocre. En 1985, sous l'ère de Deng Xiaoping (1904-1997), ces «médecins aux pieds nus 》 ont été amenés à passer un examen pour acquérir le titre de «médecin de village » (xiangcun yisheng) ${ }^{5}$. Ceux ne passant pas l'examen ou échouant à celui-ci, perdaient alors le titre de médecin pour celui de «travailleur des soins primaires» (weishengyuan) [7]. Au cours des années 1990 puis des années 2000, une multiplication des règles administratives et un renforcement des régulations portant sur ces «médecins de village » ont été émis par le gouvernement Central et les gouvernements provinciaux ${ }^{6}$. La fonction de «santé publique » qui était endossée par les «médecins aux pieds-nus », était cependant laissée de côté durant cette période [8]. Le choc sanitaire causé par la première épidémie de SARS-CoV (severe acute

Vignette (๔ Carine Milcent, hopital universitaire de Hong-Kong Shenzhen)

1 Banque mondiale: https://data.worldbank.org/indicator/SH.XPD.CHEX. GD.ZS? locations $=\mathrm{CN}$

${ }^{2}$ Le $1^{\text {er }}$ octobre 1949, Mao Ze Dong proclame à Pékin la République populaire de Chine. II cumule alors les fonctions de président du Parti communiste chinois et de président de la République jusqu'à son décès en 1976.

3 Source: Banque mondiale _ https://data.worldbank.org/indicator/SP.RUR.TOTL. ZS?locations $=\mathrm{CN}$

\footnotetext{
${ }^{4}$ Ce système est souvent traduit en anglais par the county-township-village threetier health care system.

${ }^{5}$ La première initiative en ce sens date de 1979 sous une forme moins contraignante. 6 Sans être exhaustif, citons Practicing Physician Law (zhiye yishi fa) en 1999 et 'Regulations on Village Doctors Practice' (xiangcun yisheng congye guanli tiaoli) en 2003.
} 
respiratory syndrome-coronavirus), en 2003, a conduit à redonner aux « médecins de village » la fonction de prévention qu'ils avaient perdue mais qui devenait nécessaire.

Les réformes économiques du début des années 1980 avaient changé les conditions de pratique de ces médecins. Durant la période de Mao, le financement des centres communautaires de santé, incluant les « médecins aux pieds nus», était assuré par le revenu collectif des produits de la terre de chaque communauté de villages. II s'agissait donc d'un financement décentralisé. Avec la mise en place en 1986 du «système de responsabilité des ménages ${ }^{7}$, la contribution des membres des villages au système communautaire médical était devenue volontaire, provenant de la part résiduelle de leurs revenus. Face au niveau de qualité des soins supérieure des établissements hospitaliers, les individus qui en avaient les moyens ont progressivement délaissé les centres communautaires de santé et n'ont donc plus participé à leur financement. Le développement des infrastructures routières et ferroviaires, facilitant les déplacements vers les grands centres, a accéléré ce phénomène. Ainsi, le financement des centres communautaires s'est réduit, entraînant leur effritement, voire leur disparition. Les médecins de ces structures optèrent alors pour un exercice privé de leur pratique, ou mixte (public et privé). Le système de santé chinois est ainsi passé d'un système universel d'accès aux soins à un marché des soins dont les prix étaient davantage régis par la loi de l'offre et de la demande. De ce fait, les «médecins aux pieds-nus » devenus «médecins de village » à l'activité privée ou mixte, n'ont plus accepté d'inclure dans leur activité un temps de pratique pour la prévention de la santé sans y associer une rémunération adaptée.

Pour les médecins des zones rurales, le niveau de qualification est aujourd'hui défini par le Rural Doctor Practice Regulation édité en $2004^{8}$. Il est ainsi estimé que plus de $95 \%$ des médecins ruraux ont un niveau d'éducation tel que requis par le gouvernement, qui correspond au niveau collège, suivi d'une sommaire formation médicale [9], soit un minimum de 3 années au-delà du cycle primaire. Les compétences de ces médecins soulèvent cependant des questionnements sur leur adéquation avec la pratique à laquelle ils sont confrontés [10].

En fait, trois niveaux de formation existent pour les médecins. Le premier, le plus faible, est celui que nous venons de décrire pour les médecins ruraux; le deuxième niveau de formation correspond, comme pour le premier niveau, à trois années de spécialisation, mais après le lycée ; le troisième niveau correspond à un niveau de doctorat, comprenant 5 années d'études après le baccalauréat suivies de trois années en résidence hospitalière [11-13]. En 2002, $28 \%$ des médecins avaient un niveau doctorat. Cette proportion s'élevait en 2014 à $48 \%$ des médecins [14]. La formation des médecins reste donc fortement hétérogène. Ceux qui se destinent à pratiquer dans les grands hôpitaux universitaires se distinguent par une formation de médecine plus complète. Ce système de formation à plusieurs niveaux permet toutefois d'assurer la présence de médecins sur l'ensemble du territoire chinois, y compris dans les zones peu attractives, qui seront occupées par des médecins de faible niveau

7 Ce système, qui peut être considéré comme un système de bail, permet au paysan d'être rémunéré pour sa production. L'usage et la gestion des terres peuvent être alloués à des individus ou à des unités qui doivent fournir un quota ou payer une taxe fixe, mais qui peuvent disposer librement du revenu résiduel.

${ }^{8} \mathrm{http}: / /$ www. lawinfochina.com/display. aspx? lib=law\&id=3060\&CGid = \&EncodingName=big5 de formation. Un médecin dit «médecin rural » ne peut pratiquer en zone urbaine, son niveau de formation ne le permettant pas. Le médecin universitaire, quant à lui, bénéficie plus fréquemment d'une formation continue. Le niveau de formation et de compétence des médecins est ainsi explicitement défini par la structure hospitalière dans laquelle ils exercent leurs métiers.

Aujourd'hui, au-delà des dispensaires et des centres communautaires de santé, les médecins exercent dans trois grands types de structures hospitalières classées en niveaux 9 . Les hôpitaux de niveau 1 correspondent à des hôpitaux de moins de 100 lits. Les établissements hospitaliers de niveau 2 comptent entre 100 et 500 lits. Les hôpitaux de niveau 3 en comptent plus de 500 lits; ceux-ci correspondent aux centres hospitaliers universitaires et de recherche comme on les connait en France (CHU). Les médecins sont, en majeure partie, salariés de la structure dans laquelle ils exercent, que ce soit pour leur fonction publique ou en secteur privé. Les structures de petites tailles et ayant peu d'équipements et pas ou peu de lits peuvent être publiques ou privées. En revanche, les grands hôpitaux sont tous publics. Dans ces grands établissements de santé, les médecins ont un statut de fonctionnaire (bian zhi) ${ }^{10}$. Ayant des obligations envers le Parti communiste et la structure hospitalière, ces médecins entretiennent une double carrière: l'une au sein de l'appareil communiste, l'autre à l'hôpital. Ces deux carrières, qui s'autoalimentent, sont vues comme un avantage considérable [3].

Dès 1991, le gouvernement chinois a favorisé la mise en place de structures privées. Toutefois, la règle qui contraint les médecins à choisir leur structure professionnelle (un médecin ne peut partager son activité professionnelle entre une structure privée et une structure publique) implique des difficultés de recrutement dans ces structures à caractère privé. II n'est pas possible pour un médecin, à de rares exceptions près, de quitter le secteur public pour le secteur privé et d'y revenir ensuite. Cette règle a été assouplie en 2017 mais reste néanmoins un frein. Le secteur hospitalier privé se cherche donc et peine à attirer les médecins qui sont

\footnotetext{
9 Des subdivisions existent dans chaque niveau. Notons que le niveau 3 est chapeauté par le niveau d'excellence appelé 3AAA, correspondant aux hôpitaux offrant le meilleur niveau d'équipements en plus d'un niveau d'excellence de qualification et de compétence du personnel médical.

10 Les fonctionnaires en République populaire de Chine peuvent faire partie des « unités institutionnelles » (shi ye bu men), comme pour ceux des hôpitaux publics. Les postes, salaires et budgets de ces unités sont définis et (en général) alloués (totalement ou partiellement selon les cas) par le gouvernement. Ce système d'allocation s'appelle Bian zhi. De façon générale, ce terme signifie « organisation du travail », «structure du personnel » et, par extension, peut désigner le personnel permanent, celui qui est bien planifié à l'intérieur de la structure. Utiliser ici Bian zhi est un abus de langage qui désigne le contenu par le contenant, un abus de langage courant, notamment lors de conférences mêlant membres des institutions étatiques et personnels hospitaliers et académiques (médecins/chercheurs).
} 
qualifiés pour exercer dans les hôpitaux de type «universitaire ». II se trouve dans l'obligation de recruter des médecins soit insuffisamment qualifiés pour être recrutés dans ces hôpitaux de niveau 3, soit arrivés en fin de carrière. Dans les zones péri-urbaines et rurales, les structures à l'origine publiques se sont progressivement privatisées ou sont devenues mixtes, avec pour conséquence, une augmentation considérable du coût des soins.

\section{Une demande de soins qui s'est concentrée}

En Chine, accès aux soins rime avec accès à l'hospitalisation en établissement public, au détriment des autres possibilités de soins. Ces établissements emploient en effet $64 \%$ des médecins diplômés, s'occupent des soins hospitaliers et d'une part importante des soins externes ; $82 \%$ des patients y sont admis. Par ailleurs, $40 \%$ des soins que l'on appelle soins «de ville » en France, sont prodigués dans ces structures qui concentrent la moitié des dépenses de soins [15].

Comment la Chine en est-elle arrivée à cette situation ? En premier lieu, le prix des soins a explosé. Pour diverses raisons plus ou moins liées aux réformes économiques, le système communautaire d'accès aux soins s'est délité, et si l'organisation en trois niveaux perdure, l'accès aux soins n'est plus gratuit. Le poids des dépenses de santé pour les familles a augmenté de façon exponentielle depuis les années 1980. Cela a conduit, au début des années 2000, pour une part croissante de la population, à des incapacités de paiement. Le tournant a été le choc de la crise sanitaire causé par l'épidémie due au virus SARS-CoV. La gestion de l'épidémie en 2003 a en effet accéléré la mise en place de systèmes d'assurances publiques de santé, remplaçant le système de gratuité qui s'était évaporé durant les vingt années séparant le SRAS des réformes économiques. Durant la première décennie des années 2000, trois types de système d'assurance publique de santé ont été mis en place, selon la localisation (rurale ou urbaine) et le statut (migrants ou résidents urbains) des individus. Ces assurances sont gérées au niveau local et le panier des soins pris en charge varie avec leur type et la localité concernée. En dix ans, ces assurances publiques de santé, conseillées mais volontaires, sont parvenues à couvrir $95 \%$ de la population chinoise. Toutefois, les restes à charge demeurent très importants. II n'y a pas de transférabilité entre les trois caisses d'assurance ${ }^{11}$. Ainsi, un travailleur migrant ayant contracté une assurance de sa zone rurale ( $\mathrm{New}$ rural Cooperative Medical Scheme, NCMS) et travaillant dans une zone urbaine, ne sera remboursé de ses soins que s'ils lui sont prodigués dans sa localité d'origine. En 2014, selon Sivadasan et Zhang, comparativement à un résident urbain ayant souscrit une assurance publique (Urban Resident-based Basic Medical Insurance, URBMI) pour qui les dépenses médicales seront couvertes, en moyenne à $80 \%$, le travailleur migrant devra payer cinq fois plus de reste à charge malgré un revenu deux fois moins important [16]. La même année, le taux moyen de remboursement était de 50 \% pour les hôpitaux provinciaux et de $85 \%$ pour les hôpitaux préfectoraux [17]. De ce fait, les travailleurs migrants ont le choix entre

\footnotetext{
11 Malgré des séries de réformes dont, en 2018, la création du National Healthcare Security Administra-
} tion pour la fongibilité de ces caisses, cette situation très inégalitaire perdure encore. des soins très coûteux dans leur localité de travail, en accédant à des hôpitaux d'excellence, et un retour dans leur lieu de naissance, à un coût inférieur mais dans des structures hospitalières de moindre qualité.

Jusqu'aux années 1980, la demande de soins était encadrée et canalisée : la population était d'abord prise en charge localement puis redirigée en fonction des besoins ; il n'y avait pas le choix de la structure, du dispensaire à l'hôpital universitaire. Avec les réformes économiques introduites par Deng Xiao Ping, la population s'est fortement enrichie, avec de très fortes inégalités. Sur le plan économique, le produit intérieur brut (PIB) chinois talonne aujourd'hui celui des États-Unis ${ }^{12}$. Le PIB par habitant était de 310 dollars en 1989 . Trente ans plus tard (en 2019), il atteint 10260 dollars $^{13}$ (environ 8 900 euros). Dans le même temps, le système de santé s'est privatisé et est devenu flexible, les patients pouvant se rendre dans la structure de leur choix. La demande s'est donc concentrée dans les établissements universitaires, des structures offrant une plus grande qualité de soins. La structuration en entonnoir de l'accès aux soins n'est donc plus de mise aujourd'hui. Les patients qui en ont la possibilité privilégient l'accès aux hôpitaux de niveau 3 , où la qualité des soins est administrativement définie comme haute. Un nombre croissant de patients ont ainsi eu la possibilité de se rendre dans ces hôpitaux d'excellence qui sont désormais submergés par la demande de soins, avec un temps de consultation de deux minutes en moyenne [18]. Les médecins, débordés par le nombre de patients, sont exténués, et les patients frustrés : un cocktail qui peut aller jusqu'à une violence physique vis-à-vis du personnel hospitalier, directement ou par l'intermédiaire d'hommes de main (yi nao) employés par les patients, d'où la présence dans les complexes hospitaliers d'un poste de police assurant la sécurité des personnels soignants.

Différentes réformes concomitantes ou successives ont été mises en place pour juguler cette affluence. Parmi celles-ci, la multiplication des centres de santé, la volonté d'une formation spécifique pour les médecins généralistes, la mise en place d'un système électronique de files d'attente pour éviter les effets d'affluence dans les halls d'accueil hospitaliers, et le développement de consultations à distance. C'est ce dernier instrument qui est aujourd'hui favorisé pour relâcher la contrainte sur l'offre de soins universitaires et qui transforme le système de santé chinois en un système de santé tout à fait original.

\footnotetext{
12 D'après la Banque mondiale, le PIB chinois en 2019 est de 14,34 milliards de dollars, en seconde place après les États-Unis, avec un PIB de 21,43 milliards de dollars.

13 D'après la Banque mondiale.
} 


\section{L’hôpital public}

Le statut « public » des établissements de santé chinois nécessite d'être précisé. Au début des années 1980, l'État central finançait, en moyenne, $60 \%$ des dépenses des établissements hospitaliers. En 1992, le ministère de la Santé chinois a officiellement accordé une plus grande autonomie aux hôpitaux publics. II leur a été permis de facturer leurs services et de réaliser des profits. En 2003, la part de financement public s'était ainsi réduite à $7 \%$, en moyenne. Aujourd'hui, les établissements publics sont responsables de leurs pertes et de leurs dettes, mais, en compensation, ils conservent le potentiel surplus généré par leur activité. L'hôpital public en République Populaire de Chine a donc ceci de particulier qu'il reste de statut public tout en ayant une gestion de type privée : il agit désormais comme une entreprise, maximisant son profit, fixant ses prix, définissant ses choix d'investissements, tout en ayant un mode de gouvernance traditionnelle d'institution publique. Les établissements peuvent aussi s'associer à des structures privées en créant des jointventures et investir dans un département de santé privé à but lucratif établi au sein de la structure hospitalière publique. Des compléments de revenus, aux règles opaques, sont également distribués aux médecins et aux personnels hospitaliers; ces compléments dépendent de l'activité à but lucratif de l'établissement public. Ce schéma s'ajoute à une pratique déjà courante de pots-de-vin [19].

Pour limiter le profit de ces établissements, des réformes ont donc été mises en place, avec, par exemple, le mark-up zero, qui correspond à l'absence de marge bénéficiaire [20]. De même que les hôpitaux, les dispensaires sont ciblés par cette réforme du mark-up zéro. Ils ont également dû adopter le catalogue pharmaceutique de base (basic pharmaceutical catalogue, jiben yaowu mulu) pour prescrire en priorité les produits de santé sans profit. Des ruptures organisées de stocks de médicaments permettent cependant de contrecarrer ces contraintes, favorisant les médicaments plus rentables pour la structure. Plus récemment, une autre réforme a intégré dans les établissements hospitaliers le paiement par groupe homogène de séjour (GHS) ${ }^{14}$ [21], qui fait écho à la tarification à l'activité (T2A) appliquée en France. Ces différentes mesures ont cependant leurs limites. II en est de même concernant le phénomène de sur-prescription. En effet, pour distinguer les actes et procédures nécessaires de ceux réellement superflus, il est nécessaire d'avoir un système d'information généralisé et efficace ainsi qu'une confiance quant à l'utilisation faite des données. Ces paramètres n'étant pas réunis, les établissements publics sont réticents à partager leurs données.

Tous ces éléments expliquent les controverses apparues depuis 2003 sur la portée de ces réformes. L'option offerte par les géants d'internet, les équivalents chinois des GAFAM ${ }^{15}$, apparaît à l'ensemble du système comme plus viable, autant en termes de maîtrise du circuit des données (utilisant le nuage ou cloud), et leur utilisation intelligente par intelligence artificielle, que de secret de l'information.

\footnotetext{
14 Les GHS sont des unités de mesure qui prennent en compte, pour chaque patient, la maladie traitée, le mode de prise en charge, les soins prodigués. À chaque GHS correspond un tarif, qui est déterminé chaque année par le ministère de la Santé.

15 Google, Apple, Facebook, Amazon et Microsoft.
}

Sur ce dernier point, des accords de coopération permettent de contractualiser la transmission des données.

\section{Un système de santé émergent}

Les applications proposées par les géants chinois, les BATX ${ }^{16}$, offrent un service qualifié « d'hôpital virtuel », avec des options, telles que des consultations en ligne, la supervision médicale, des forums de communautés sur la santé, des rendez-vous pour des soins externes, et des services de soins hospitaliers à domicile. Ces services se sont multipliés, avec une régulation qui reste néanmoins à établir. En effet, les téléconsultations et télé-expertises entrent dans une zone «grise»: il ne s'agit pas d'activités professionnelles établies dans une structure sanitaire privée, mais d'activités «tolérées». Des expérimentations ont été lancées pour autoriser officiellement ces pratiques ${ }^{17}$. Cette offre virtuelle permet aux patients d'accéder à des médecins hautement qualifiés, voire réputés, ce qui n'est pas possible en présentiel ou uniquement avec des délais bien trop considérables $[22-26]^{18}$.

De façon schématique, l'offre digitale intègre 1) le consommateur et son accès facilité aux soins, 2) le fournisseur de soins, qui est alors virtuellement assisté, et 3) la coordination du système de santé dans son ensemble.

Pour le patient, le digital lui offre des informations médicales à travers des plateformes de discussions entre patients ainsi que des plateformes d'information médicale. Les objets connectés lui permettent d'être surveillé à distance ou de s'auto-surveiller. Il s'agit, par exemple de trackers de fitness, des montres déterminant le niveau d'activité, ou de détecteurs/capteurs (pouvant lancer des alertes en cas de problèmes). En cas de maladies, notamment chroniques, ces outils permettent une surveillance de fonctions précises à distance, une meilleure observance médicale par l'envoi de rappels, des thérapies et certaines interventions par voie numérique, des conseils et consultations à distance auprès de praticiens ayant un haut niveau de compétence, sans subir les temps d'attente en présentiel dans les structures où ces médecins exercent, et

\footnotetext{
16 Baidu, Alibaba, Tencent et Xiaomi. En santé, J.D. Com est également à ajouter à cette liste.

17 Chinadaily. Internet-based healthcare helps fight against epidemic. https://www. chinadaily.com.cn/a/202003/04/WS5e5efcb9a31012821727c175.html et, https://www. spglobal.com/marketintelligence/en/news-insights/latest-news-headlines/china-sbig-tech-to-continue-disruption-of-healthcare-sector-despite-crackdown-61610882 18 Milcent C. From conventional healthcare to e-health: Social and Spatial Transformation:Using a comparison between HK and mainland China. Journal of Clinical and Translational Research, à paraître.
} 
finalement la possibilité d'achat de médicaments, dont ceux prescrits en ligne.

En ce qui concerne les fournisseurs de soins, ils bénéficient d'un support de décision clinique apporté par l'intelligence artificielle, le stockage d'informations dans des nuages, et la consultation d'informations à partir de big data, des dispositifs intelligents, tels que de la robotique médicale ou des outils intelligents (stéthoscopes, équipements d'imagerie, etc.), des outils de formation, dont des outils de simulation en réalité virtuelle/réalité augmentée/réalité mixte.

Pour le système de soins, le digital apporte des outils afin d'éviter les files d'attente de patients venant engorger l'organisation hospitalière, grâce à des plateformes de prise de rendez-vous, des portails de santé pour les patients, et par la mise en place de systèmes d'information hospitaliers ainsi que de dossiers médicaux et de santé électroniques, pour un meilleur suivi de l'activité et des besoins de soins. Le digital permet également l'administration de la facturation médicale, d'avoir accès à des logiciels de télémédecine et à des outils de vidéoconférence et permet des transferts d'informations sur les patients.

Le système de soins chinois est confronté au manque d'attractivité des centres de santé communautaires et des petites structures hospitalières. Le niveau de qualité offert n'atteint pas celui désormais attendu par les patients. La digitalisation du système d'offre de soins ouvre ainsi des opportunités pour combler l'insuffisance d'offre de qualité et limiter le niveau du coût des dépenses, insuffisamment pris en charge par les assurances publiques. Elle permet de désengorger les structures publiques de haute qualité - tout en permettant des dépenses de soins à moindre coût - ainsi qu'une prise en charge des individus par des assureurs privés profitant du contrôle des coûts des réseaux de soins associés à ces applications. II s'agit d'un système de santé dont le cœur pourrait être régi par les géants de l'internet.

Les autorités chinoises naviguent entre un fort encouragement à l'esystème de soins et un contrôle des géants d'internet qui s'imposent sur le marché. À titre d'exemple, l'État chinois a multiplié dernièrement les amendes anti-trust envers des filiales santé de géants d'internet, comme Ant Group, une filiale d'Alibaba, l'équivalent d'Amazon aux États-Unis. Ce système nécessite encore un contrôle et une régulation afin d'assurer une confidentialité de l'information et une éthique des soins. Avec la crise sanitaire causée par la pandémie de COVID-19 (coronavirus disease 2019), due au SARS-CoV-2, la place du digital dans le système de soins chinois est devenue centrale. Les aspects inégalitaires entraînés par ce nouveau système de soins sont donc plus que jamais à étudier. $\diamond$

The Chinese health system

\section{LIENS D'INTÉRÊT}

Les auteurs déclarent n'avoir aucun lien d'intérêt concernant les données publiées dans cet article.

\section{RÉFÉRENCES}

1. Hesketh T, Wu D, Mao L, Ma N. Violence by patients against doctors is on the increase in China, 2012. BMJ $2012 ; 345$ : e5730.

2. Cai R., Tang J, Deng C, et al. Violence against health care workers in China, 2013-2016: evidence from the national judgment documents. Hum Resour Health 2019; $17: 103$.
3. Milcent C. Medical staff. In : Milcent C (ed). Healtchare reform in China: from violence to digital healthcare. Palgrave Macmillan, 2018.

4. Gross M. Farewell to the god of plague. chairman mao's campaign to deworm China. University of California Press, 2016.

5. Xiaoping F. Barefoot doctors and western medicine in China. University of Rochester Press, 2015.

6. Liu X, Wang J. An introduction to China's health care system. J Public Health Policy $1991 ; 12$ : 104-16.

7. Decheng L. Chuangzao yu chonggou: jitihuashiqi nongcun hezuo yiliao zhidu he chijiao yisheng xianxiang yanjiu (creation and restructuring: research on the rural collective medical scheme in the collective era and the barefoot doctor phenomenon). Beijing : China Book Press, 2013.

8. Tu J. The lost generation barefoot doctors in post-reform china. In : The health system and access to healthcare in China. China Perspective 2016 ; (numéro special) : 4. http://www.cefc.com.hk/issue/chinaperspectives-20164/

9. Li H, Wang Z, Jiang $\mathrm{N}$, et al. Lifelong learning of Chinese rural physicians: preliminary psychometrics and influencing factors. BMC Med Educ 2015 ; $15: 192$.

10. Li X, Shen JJ, Yao F, et al. Does exam-targeted training help village doctors pass the certified (assistant) physician exam and improve their practical skills? A cross-sectional analysis of village doctors' perspectives in Changzhou in Eastern China. BMC Med Educ 2018; 18 : 107.

11. Wu L, Wang $Y$, Peng $X$, et al. Development of a medical academic degree system in China. Med Educ Online 2014 ; 19. https://doi.org/10.3402/ meo.v19.23141

12. Zhu J, Li W, Chen L. Doctors in China: improving quality through modernisation of residency education. Lancet 2016; 10054 : 1922.

13. Hou J, Michaud C, Li Z, et al. Transformation of the education of health professionals in China: progress and challenges. Lancet 2014 ; 384 : 819-27.

14. Hsieh CR, Tang C. The multi-tiered medical education system and its influence on the health care market - China's flexner report. Hum Resour Health $2019 ; 17: 50$.

15. National Health Commission. China national health statistical yearbook 2003-2018. Peking Union Medical College Press, 2018.

16. Sivadasan $\mathrm{K}$, Zhang C. Improving healthcare insurance for migrant workers in China. Master of public administration in international development. John F. Kennedy School of Government, Harvard University, 2014.

17. Ma J, Xu J, Zhang Z, Wang J. New cooperative medical scheme decreased financial burden but expanded the gap of income-related inequity: evidence from three provinces in rural China. Int J Equity Health 2016 ; $15: 72$.

18. Jin $G$, Zhao Y, Chen $C$, et al. The length and content of general practice consultation in two urban districts of Beijing: a preliminary observation study. PLoS One $2015 ; 10$ : e0135121.

19. Shun Ching Chan C. A market of distrust: unofficial payments for hospital care in China. The $12^{\text {th }}$ Conference of the European Sociological Association (ESA), 2015

20. Du J, Xie J, Qian Y, et al. Impact of China's zero mark-up drug policy on drug cost of NCDs' outpatients: an interrupted time series analysis. BMC Health Serv Res $2021 ; 21: 404$.

21. Zhang L, Sun L. Impacts of diagnosis-related groups payment on the healthcare providers' behavior in china: a cross-sectional study among physicians. Risk Manag Healthc Policy 2021 ; 14 : 2263-76.

22. Tu J, Wang C, Wu S. The internet hospital: an emerging innovation in China. Lancet Glob Health 2015 ; 38 : e445-6.

23. Guidelines on promoting Internet plus Health care services during Covid19 prevention and control (in Chinese). http://www.gov.cn/zhengce/ zhengceku/2020-03/03/content_5486256.htm

24. Sun $S, Y_{u}$ K, Xie Z, et al. China empowers internet hospital to fight against Covid-19. J Inf Secur 2020; 811 : e67-8.

25. Lee GH, Seong NY. Application of artificial intelligence-based technologies in the healthcare industry: opportunities and challenges. Int J Environ Res Public Health 2021 ; 18 : 271.

26. Sun S, Xie Z, Yu K, et al. Covid-19 and healthcare system in China: challenges and progression for a sustainable future. Global Health 2021 ; $17: 14$.

evidence from the national judgment documents. Hum Resour Health $2019 ; 17: 103$.

TIRÉS À PART

C. Milcent 